\title{
A Lie Theoretic Galois Theory for the Spectral Curves of an Integrable System: I
}

\author{
Andrew McDaniel ${ }^{1}$ and Lawrence Smolinsky ${ }^{2 \star}$ \\ ${ }^{1}$ Department of Mathematics, George Mason University, Fairfax, VA 22030, USA \\ 2 Department of Mathematics, Louisiana State University, Baton Rouge, LA 70803, USA
}

Received October 7, 1991

\begin{abstract}
In the study of integrable systems of ODE's arising from a Lax pair with a parameter, the constants of the motion occur as spectral curves. The specific curves depend upon the representation of the Lie algebra. In this paper a Galois theory of spectral curves is given that classifies the spectral curves from an integrable system. The spectral curves correspond to conjugacy classes of certain subgroups of the Weyl group for the Lie algebra. The theory is illustrated with the periodic Toda lattice.
\end{abstract}

\section{Introduction}

One mechanism for producing constants of the motion for completely integrable systems is the Lax pairing. The idea introduced by Peter Lax was to express systems of differential equations in the form $\frac{d A}{d t}=[A, B]$. For finite dimensional systems the following desirable situation often occurs:

1. $A$ and $B$ lie in a Lie algebra $\mathfrak{g}$,

2. $A$ and $B$ are functions of time $t$ and rational functions of a parameter $s$ where $s$ is a coordinate on an algebraic curve $P$.

For each representation $\rho$ of $\mathfrak{g}$, the characteristic polynomial of $\rho(A)$ defines a curve by the equation $0=\operatorname{det}(\rho A(s, t)-z)$. It is a consequence of the Lax form of the differential equation for $A$ that $\{(s, z) \mid 0=\operatorname{det}(\rho A(s, t)-z)\}$ is independent of time for any representation $\rho$. The curves defined by $0=\operatorname{det}(\rho A(s, t)-z)$ are in general reducible and the irreducible components of these various curves are called spectral curves. These curves are equipped with projections to $P$ via the $s$ coordinate. This arrangement has been used by many mathematicians to examine completely integrable systems, e.g., van Moerbeke and Mumford [vMM]; Adler and van Moerbeke [AvM1, 2]; McDaniel [Mc]; Kanev [K]; Griffiths [G];

\footnotetext{
* Partially supported by a Louisiana Education Quality Support Fund grant LEQSF (8789)-RD-A-8
} 
Bobenko, Reyman and Semenov-Tian-Shansky [BRS]; Zakharov and Mikhailov $[\mathrm{ZM}]$. A spectral curve is important not only as providing the constants of the motion of a completely integrable system, but also because the flow of the system often linearizes on the Jacobi variety of the curve. Moreover the results of this paper suggest that given a matrix Lax equation with a parameter, an underlying Lie algebra could be revealed via its Weyl group from the Galois theory of the spectral curve.

This paper provides a Galois theory for the family of spectral curves with their projections that arise as constants of the motion from a Lax equation with a parameter. The main result (Theorems 13 and 15) is that the spectral curves for a given initial condition can be indexed by certain subgroups of the Weyl group. This paper treats the spectral curves themselves. It does not deal with the question of linearization on the Jacobians of the spectral curves as in [G] and [K]. Such questions will be dealt with in the sequel.

The method of proof is to construct large spectral varieties $\tilde{E}_{\lambda}$ which sit over the Lie algebras themselves (whereas a spectral curve sits over the parameter space). The spectral curves are then irreducible components of the pullbacks of

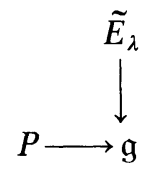

The spectral varieties have both a Lie theoretic description via the adjoint map and a more concrete description as eigenvalue covers. The key lemma is Lemma 3 displaying an isomorphism between these two descriptions, which leads to a Galois theory for the spectral varieties in terms of conjugacy classes of parbolic subgroups of the Weyl group (Theorem 4). This theory is pulled back to the spectral curves (Theorem 13) and applied to the periodic Toda lattice (Proposition 20) as a specific example.

The paper proceeds as follows. After the notation is established, the section spectral varieties contains the basic construction of a spectral variety. The following section classification of the spectral varieties contains the classification theorem of spectral varieties. This section examines spectral varieties from a purely Lie algebraic viewpoint. In stabilizers of weights, an analysis for the infinite families of algebras as well as types $G_{2}$ and $F_{4}$ is given. The classification of the spectral varieties is related to subdiagrams of the various Dynkin diagrams. The section spectral curves relates spectral curves to spectral varieties and the classification theorem for spectral curves is given (Theorem 13). It is a corollary that a Lax equation with an initial condition is associated to only finitely many spectral curves. The final section, the Toda lattice, contains the detailed example of the Toda lattice for the infinite families of algebras. The Galois theory for spectral curves is studied for generic initial conditions. The Galois group of the splitting field of the defining polynomial of the spectral curve over the field of meromorphic functions on the Riemann sphere is shown to be the Weyl group for a generic initial condition. This gives a minor correction to [AvM1].

In [AvM1] and [AvM2], Adler and van Moerbeke use the KostantAdler-Symes theorem [AvM1, p. 282] and the van Moerbeke-Mumford linearization process $[\mathrm{vMM}]$ to examine several completely integrable systems via spectral curves with $P$ the Riemann sphere. They conclude that the spectral curves are 
related by' algebraic correspondences. This paper can be viewed as an extension of that result.

The authors are grateful to William Adkins for a discussion concerning the relevant analytic geometry and to Mark Adler for reading a preliminary version of this paper.

\section{Notation}

The following common notation is used throughout this paper. The letter $g$ denotes a complex simple Lie algebra. A Cartan subalgebra is fixed and denoted $\mathfrak{h}$. The corresponding connected Lie groups are denoted $G$ and $H$ respectively. The adjoint group may be taken as $G$, but the particular connected Lie group which is used is not consequential as this paper is concerned with $G / H$ rather than $G$ or $H$ individually. Let $N_{H}$ and $C_{H}$ denote respectively the normalizer and the centralizer of the maximal torus $H$ in $G$. Since $g$ is simple, $C_{H}=H$. The Weyl group $W$ is the quotient group $N_{H} / C_{H}$, which acts on $\mathfrak{h}$ by $n C_{H} \cdot h=\operatorname{Ad}_{n} h$. Weights for the pair $(\mathfrak{g}, \mathfrak{h})$ are denoted by $\lambda$ or by $\lambda$ and $\gamma$ if two of them are under discussion.

The subsets of regular elements of $\mathfrak{g}$ and $\mathfrak{h}$ are denoted respectively by $\mathfrak{g}_{*}$ and $\mathfrak{h}_{*}$. One convenient characterization of the regular elements of $\mathfrak{g}$ is as those elements which are contained in a unique Cartan subalgebra. Other elements either lie in no Cartan subalgebra or in infinitely many of them. A different characterization shows that the regular elements form a quasiaffine variety within either $\mathfrak{g}$ or $\mathfrak{h}$. Form the characteristic polynomial $\operatorname{det}\left(a d_{x}-z\right)$ of the Lie algebra $\mathfrak{g}$. When expanded in powers of $z$, there is a lowest coefficient which does not vanish identically. The regular elements are those $x$ for which this coefficient is non-zero. Since these coefficients are polynomials in $x \in \mathfrak{g}$, the regular elements are the complement of the zero set of a polynomial. This paper looks at branched covers over $\mathfrak{g}$ which are unbranched over $\mathfrak{g}_{*}$. The portion of a cover $E$ which lies over $\mathfrak{g}_{*}$ will be denoted $E_{*}$.

\section{Spectral Varieties (Eigenvalue Covers)}

This section presents the spectral varieties in concrete guise; namely, a spectral variety is an irreducible component of the variety defined by the characteristic polynomial of a matrix representation of a Lie algebra.

Definition. Let $\rho$ be a finite dimensional irreducible representation of $\mathfrak{g}$. Define $\mathscr{E}_{\rho} \subset \mathfrak{g} \times \mathbf{C}$ to be the variety defined by the equation $\operatorname{det}(\rho(x)-z)=0$.

Since $\mathfrak{g}$ is isomorphic to $\mathbf{C}^{N}$ as a complex vector space and $\rho$ is linear, $\operatorname{det}(\rho(x)-z)$ is a polynomial for any representation. The variety $\mathscr{E}_{\rho}$ is in general reducible and decomposes via the dominant weights. These pieces are independent of the representation in that they depend only on the weights.

Proposition 1. For each dominant weight $\lambda$ there is a polynomial $p_{\lambda}(x, z)$ such that if $\rho$ is any finite dimensional representation and the multiplicity of the weight $\lambda$ in $\rho$ is $m_{\lambda}$ then

$$
\operatorname{det}(\rho(x)-z)=\prod_{\lambda}\left(p_{\lambda}(x, z)\right)^{m_{\lambda}},
$$

where $\lambda$ runs through the dominant weights. 
Proof. For each $x \in \mathfrak{g}$ let $x=x_{s}+x_{n}$ be its decomposition into commuting semisimple and nilpotent parts. Let $\mathfrak{h}$ be the Cartan subalgebra in terms of which the weights are defined. The element $x_{s}$ is in some Cartan subalgebra so there is a $g \in G$ such that $\operatorname{Ad}_{g} x_{s} \in \mathfrak{h}$. Let

$$
p_{\lambda}(x, z)=\prod_{\gamma \in W \cdot \lambda}\left(\gamma\left(\operatorname{Ad}_{g} x_{s}\right)-z\right)
$$

where $W \cdot \lambda$ denotes the orbit of the weight $\lambda$ under the action of the Weyl group. This expression for $p_{\lambda}(x, z)$ does not depend on the choice of $g$ since the ambiguity is up to conjugation by an element of the Weyl group. Since $x_{s}$ and $x$ have the same eigenvalues with multiplicities, $\operatorname{det}(\rho(x)-z)=\operatorname{det}\left(\rho\left(x_{s}\right)-z\right)$ and $\operatorname{det}(\rho(x)-z)=$ $\prod_{\lambda}\left(p_{\lambda}(x, z)\right)^{m_{\lambda}}$. It remains to see that $\rho_{\lambda}(x, z)$ is a polynomial. This can be shown by using induction on the partial ordering of the weights by levels. For a dominant weight $\lambda$, let $\rho_{\lambda}$ be the irreducible representation with highest weight $\lambda$. In $\rho_{\lambda}$ the weight $\lambda$ occurs with multiplicity one and the other weights lie in strictly lower levels. If $\lambda$ is a fundamental dominant weight then $p_{\lambda}(x, z)=\operatorname{det}\left(\rho_{\lambda}(x)-z\right)$ so $p_{\lambda}(x, z)$ is a polynomial. Now suppose $\lambda$ is any dominant weight and that $p_{\gamma}(x, z)$ is a polynomial for all dominant weights $\gamma$ at a lower level than $\lambda$. Then, $\operatorname{det}\left(\rho_{\lambda}(x)-z\right)=\prod_{\gamma \prec \lambda}\left(p_{\gamma}(x, z)\right)^{m_{i}} \cdot p_{\lambda}(x, z)$ so $p_{\lambda}(x, z)$ is a rational function. Since it takes finite values, it is a polynomial.

Definition. Let $E_{\lambda}$ be the variety defined by $p_{\lambda}(x, z)=0$.

These varieties are irreducible for each dominant weight $\lambda$. This will be proven below. Each variety $E_{\lambda}$ comes equipped with a projection pr: $E_{\lambda} \rightarrow \mathfrak{g}$ induced by $\mathfrak{g} \times \mathbf{C} \rightarrow \mathfrak{g}$. Denote the inverse image of the regular elements $\mathfrak{g}_{*}$ by $E_{\lambda *}$.

The variety $E_{\lambda}$ is in general singular. The normalization of a variety $X$ will be denoted $\tilde{X} \rightarrow X$. By composing pr: $E_{\lambda} \rightarrow \mathfrak{g}$ with normalization one obtains a morphism $\tilde{E}_{\lambda} \rightarrow \mathfrak{g}$ which will generally also be denoted pr but for the purposes of this paragraph will be denoted pr. The regular elements $\mathfrak{g}_{*}$ in $\mathfrak{g}$ form a Zariski open set so $\mathrm{pr}^{-1}\left(\mathfrak{g}_{*}\right)=E_{\lambda *} \subset E_{\lambda}$ is also a Zariski open set. Since normalization is a local process $\widetilde{\mathrm{pr}^{-1}}\left(\mathfrak{g}_{*}\right) \cong \tilde{\mathrm{pr}}^{-1}\left(\mathfrak{g}_{*}\right)$ so $\tilde{E}_{\lambda *}$ unambiguously denotes a certain Zariski open subset of $\tilde{E}_{\lambda}$. It will be shown subsequently that $\tilde{E}_{\lambda *}$ is a covering space of $\mathfrak{g}_{*}$.

The varieties $\tilde{E}_{\lambda}$ are the main objects of study. These are the varieties which yield spectral curves. If $A: \mathbf{C} \rightarrow \mathfrak{g}$ is a matrix in a parameter $s$ then the pullback of $\tilde{E}_{\lambda}$ along $A, A^{*}\left(\tilde{E}_{\lambda}\right)$ gives rise to the spectral curves (see the section titled spectral curves).

\section{Classification of the Spectral Varieties}

The bridge between the various spectral varieties is the covering space described below. Since the Cartan subgroup $H$ is a closed subgroup of $G$, both $G$ and $G / H$ are smooth quasiprojective varieties $\left[H 1\right.$, p. 80]. The space $G / H \times \mathfrak{h}_{*}$ is also a smooth quasiprojective variety.

Lemma 2. Let $\pi: G / H \times \mathfrak{h}_{*} \rightarrow \mathfrak{g}_{*}$ be given by $\pi(g H, h)=\operatorname{Ad}_{g} h$.

(a) $\pi$ is a morphism.

(b) $\pi: G / H \times \mathfrak{h}_{*} \rightarrow \mathfrak{g}_{*}$ is the projection map of a regular covering space with group of translations $W$. The action of $W$ is given by $\left(n C_{H}\right) \cdot(g H, h)=\left(g n^{-1} H, \mathrm{Ad}_{n} h\right)$. 
Proof. (a) 'The map $\pi$ is well-defined since $\operatorname{Ad}_{g h^{\prime}} h=\operatorname{Ad}_{g} \operatorname{Ad}_{h^{\prime}} h$ and $\operatorname{Ad}_{h^{\prime}} h=h$ for $h^{\prime} \in H$. The map Ad: $G \times \mathfrak{h} \rightarrow \mathfrak{g}$ is a polynomial map. Since it is constant on cosets of $H \times 0$, it factors through $G / H \times \mathfrak{h}$ by a unique morphism in accordance with the universal mapping property of quotients [H1, p. 83]. Since $\operatorname{Ad}^{-1}\left(\mathfrak{g}_{*}\right)=G / H \times \mathfrak{h}_{*}$, $\pi$ is the restriction of the morphism Ad to the Zariski open set $G / H \times \mathfrak{h}_{*}$. It follows that $\pi$ is a morphism.

(b) Let $W$ act on $G / H \times \mathfrak{h}_{*}$ by $\left(n C_{H}\right) \cdot(g H, h)=\left(g n^{-1} H, \mathrm{Ad}_{n} h\right)$. No element in $W$ except $e C_{H}$ has a fixed point since $g n^{-1} H=g H$ implies $n \in H=C_{H}$. Since $G / H \times \mathfrak{h}_{*}$ is a Hausdorff space (in the manifold topology) and $W$ is a finite group acting without fixed points, $W$ acts properly discontinuously on $G / H \times \mathfrak{h}_{*}$. Therefore $G / H \times \mathfrak{h}_{*} \rightarrow G / H \times \mathfrak{h}_{*} / W$ is a regular covering space with group of covering translations $W$. The map $\pi$ factors through $\pi^{\prime}: G / H \times \mathfrak{h}_{*} / W \rightarrow \mathfrak{g}_{*}$. The map $\pi^{\prime}$ is a continuous map of manifolds. It is one-to-one since $\operatorname{Ad}_{g} h=\operatorname{Ad}_{g^{\prime}} h^{\prime}$ implies $\operatorname{Ad}_{g^{-1}} g^{\prime} h^{\prime} \in \mathfrak{h}$ and since $h^{\prime}$ is regular this implies $g^{-1} g^{\prime} \in N_{H}$. The map $\pi$ is onto since every regular element is in a Cartan subalgebra and all the Cartan subalgebras are conjugate. The map $\pi^{\prime}$ is therefore a homeomorphism and so (b) follows.

Let $\lambda$ be a weight. Define $\pi_{\lambda}: G / H \times \mathfrak{h}_{*} \rightarrow E_{\lambda *}$ by $\pi_{\lambda}(g H, h)=\left(\operatorname{Ad}_{g} h, \lambda(h)\right)$. Since Ad is a polynomial and $\lambda$ is linear, $\pi_{\lambda}$ is a morphism. The variety $G / H \times \mathfrak{h}_{*}$ is a smooth (and hence normal) variety, so $\pi_{\lambda}$ lifts to a unique morphism $\tilde{\pi}_{\lambda}$ : $G / H \times \mathfrak{h}_{*} \rightarrow \tilde{E}_{\lambda *}$. Let $S_{\lambda}=\left\{n C_{H} \in W \mid \lambda\left(\operatorname{Ad}_{n} h\right)=\lambda(h)\right.$ for all $\left.h \in \mathfrak{h}\right\}$ so that $S_{\lambda}$ is the stabilizer of $\lambda$ under the action of $W$.

The morphism $\pi_{\lambda}$ factors through $G / H \times \mathfrak{h}_{*} / S_{\lambda}$. The factorization yields a morphism $i_{\lambda}: G / H \times \mathfrak{h}_{*} / S_{\lambda} \rightarrow E_{\lambda *}$. The morphism $i_{\lambda}$ lifts to an isomorphism $\tilde{i}_{\lambda}$ : $G / H \times \mathfrak{h}_{*} / S_{\lambda} \rightarrow \tilde{E}_{\lambda *}$. This isomorphism connects the abstract construction of spectral varieties with their concrete realizations as eigenvalue covers of $\mathfrak{g}_{*}$.

Lemma 3. The following diagram is a commutative diagram of morphisms which are covering space projections.

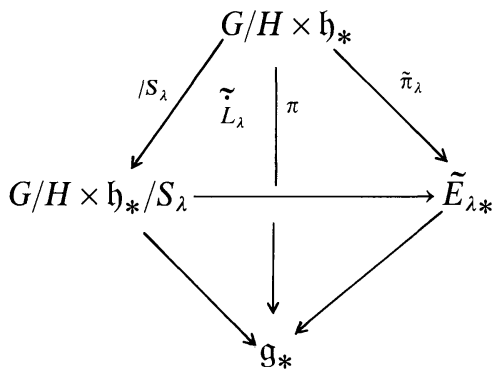

The morphism $\tilde{\pi}_{\lambda}$ is a regular covering space projection and the morphism $\tilde{i}_{\lambda}$ is an isomorphism.

Proof. Let $U \subset E_{\lambda}$ be the Zariski open subset obtained by removing the pre-image of the zero of the discriminant of $p_{\lambda}(x, z)$ and the singular set from $E_{\lambda}$. The discriminant vanishes at all those $x \in \mathfrak{g}$ such that $p(z)=p_{\lambda}(x, z)$ has a multiple root. Note that $U$ is non-empty since its image in $g$ is dense. Consider the morphism $\left.i_{\lambda}\right|_{i_{\lambda}^{-1}(U)}: i_{\lambda}^{-1}(U) \rightarrow U$. This map is clearly onto $U$. If $(\pi(g H, h), \lambda(h))=\left(\pi\left(g^{\prime} H, h^{\prime}\right)\right.$, $\left.\lambda\left(h^{\prime}\right)\right)$ then by Lemma 2 there is an $n \in N_{H}$ such that $n C_{H} \cdot(g H, h)=\left(g^{\prime} H, h^{\prime}\right)$. In particular $\operatorname{Ad}_{n} h=h^{\prime}$ so $\lambda\left(\operatorname{Ad}_{n} h\right)=\lambda\left(h^{\prime}\right)=\lambda(h)$. Since $h$ is not a member of the discriminant of $p_{\lambda}$, then $n C_{H} \in S_{\lambda}$. The morphism $\left.i_{\lambda}\right|_{i_{\lambda}^{-1}(U)}$ is a one-to-one onto 
morphism of smooth varieties and so is an isomorphism by the generalized Riemann existence theorem [Hart, p. 442]. The morphism $\tilde{i}_{\lambda}$ is thus an isomorphism on an open set and finite-to-one elsewhere. Therefore by Zariski's main theorem [Hart, p. 280] $\tilde{i}_{\lambda}$ is an isomorphism.

The diagram is commutative directly from the definition of the maps. Since $\tilde{i}_{\lambda}$ is an isomorphism and $/ S_{\lambda}$ yields a regular cover, $\tilde{\pi}_{\lambda}$ is a regular cover. The morphism $\mathrm{pr}$ is a cover since $j$ is a covering map.

Theorem 4. The spectral varieties $\tilde{E}_{\lambda *}$ are classified as varieties with projections by conjugacy classes of stabilizers of weights in the Weyl group. That is there exists an isomorphism $f: \tilde{E}_{\lambda *} \rightarrow \tilde{E}_{\gamma *}$ such that

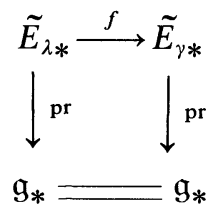

commutes if and only if $S_{\lambda}$ is conjugate to $S_{\gamma}$ in $W$.

Proof. Let $J$ be the fundamental group $\pi_{1}\left(G / H \times \mathfrak{h}_{*}\right)$. By the generalized Riemann existence theorem (proven in [GrRe] and discussed in [Hart, p. 442] or [M, pp. 40 and 118]) the algebraic covers of $\mathfrak{g}_{*}$ covered by $G / H \times \mathfrak{h}_{*}$ are classfied by conjugacy classes of subgroups of $\pi_{*}(J)$ where conjugacy is in $\pi_{1}\left(g_{*}\right)$. This is the same as the purely topological situation. Consider the exact sequence

$$
0 \rightarrow J \rightarrow \pi_{1}\left(\mathfrak{g}_{*}\right) \stackrel{q}{\rightarrow} W \rightarrow 0 .
$$

The group $\pi_{1}\left(\mathfrak{g}_{*}\right)$ acts by conjugation on this sequence. If $c_{a}$ is conjugation by $a$ then

$$
\begin{aligned}
0 \rightarrow & J \rightarrow \pi_{1}\left(\mathfrak{g}_{*}\right) \rightarrow W \rightarrow 0 \\
& \downarrow c_{a} \downarrow c_{a} \quad \downarrow c_{q(a)} \\
0 \rightarrow & J \rightarrow \pi_{1}\left(\mathfrak{g}_{*}\right) \rightarrow W \rightarrow 0
\end{aligned}
$$

commutes. Now $\pi_{1}\left(\tilde{E}_{\lambda *}\right) \subset \pi_{1}\left(\mathfrak{g}_{*}\right)$ and $\pi_{1}\left(\mathfrak{g}_{*}\right)$ acts on the collection of subsequences of $(*)$

$$
0 \rightarrow J \rightarrow \pi_{1}\left(\tilde{E}_{\lambda *}\right) \rightarrow S_{\lambda} \rightarrow 0
$$

by permuting these subsequences. Two stabilizer subgroups $S_{\lambda}$ and $S_{\gamma}$ are conjugate exactly when $\pi_{1}\left(\tilde{E}_{\lambda *}\right)$ and $\pi_{1}\left(\tilde{E}_{\gamma *}\right)$ are conjugate.

Note that $\pi_{1}\left(\mathfrak{g}_{*}\right) / J=W$ and $n \in N_{H}$ acts by taking the sequence of subgroups to

$$
0 \rightarrow J \rightarrow \pi_{1}\left(\tilde{E}_{(n \cdot \lambda) *}\right) \rightarrow S_{(n \cdot \lambda)} \rightarrow 0
$$

since covering translations change the basepoint and conjugation is the change of basepoint for the fundamental group.

Remark. Breiskorn showed that the groups $\pi_{1}\left(\mathfrak{h}_{*} / W\right)$ are generalizations of Artin's braid group [B]. The groups $\pi_{1}\left(\mathfrak{g}_{*}\right)$ are also equal to these Breiskorn braid groups [MS]. In particular, if $g$ is of type $A_{n-1}$ then $\pi_{1}\left(g_{*}\right)$ is Artin's braid group and if $g_{*}$ is of type $B_{n}$ or $C_{n}$ then $\pi_{1}\left(\mathfrak{g}_{*}\right)$ is isomorphic to the braid group of the annulus $B_{n}(\mathbf{C}-\{0\})[\mathrm{MS}]$. 
The covering spaces $G / H \times \mathfrak{h}_{*} \rightarrow \tilde{E}_{\lambda *}$ are all regular covering spaces. However, $\tilde{E}_{\lambda *} \rightarrow \mathfrak{g}_{*}$ is not.

Proposition 5. The covering space

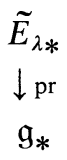

is regular if and only if $\lambda$ is a nonsingular weight or the zero weight. If $\lambda$ is a nonsingular weight then $\tilde{E}_{\lambda *} \cong G / H \times \mathfrak{h}_{*}$.

Proof. If $\lambda$ is nonsingular then $S_{\lambda}$ is trivial. These weights are precisely those that lie in the interior of a Weyl chamber. Since $\tilde{E}_{\lambda *} \cong G / H \times \mathfrak{h}_{*} / S_{\lambda}$ by Lemma 3 , $\tilde{E}_{\lambda *} \cong G / H \times \mathfrak{h}_{*}$.

If $\lambda$ is a singular weight then $S_{\lambda} \neq 1$ and by Theorem 4 , it is enough to show that $S_{\lambda}$ is not normal in $W$. If $w \in W$ then $S_{\lambda}$ and $S_{w \cdot \lambda}$ are conjugate. So if $S_{\lambda}$ is normal then every weight in the orbit is stabilized by $S_{\lambda}$. Furthermore any linear combination of weights in the orbit is stabilized by $S_{\lambda}$. If the span of an orbit is all $\mathfrak{h}$ then $S_{\lambda}$ must be trivial. But the span of an orbit is always $\mathfrak{h}$ in a simple algebra as follows. If $\mathfrak{D}$ is an orbit, $\alpha \in \operatorname{span} \mathfrak{D}$ and $\beta$ is a root then $\alpha+\frac{(\alpha, \beta)}{(\alpha, \alpha)} \beta \in \operatorname{span} \mathfrak{D}$. So take any $\beta$ with $(\alpha, \beta) \neq 0$ then $\beta \in \operatorname{span} \mathcal{D}$. But the root lattice is irreducible for the simple algebras and $W$ acts irreducibly (see $[\mathrm{H} 1,10.4]$ ). Therefore the whole root lattice is in $\operatorname{span} \mathfrak{D}$ and $\operatorname{span} \mathfrak{D}=\mathfrak{h}$.

Corollary 6. Up to isomorphism there are only finitely many $\tilde{E}_{\lambda *}$ for a given algebra g. Up to birational equivalence there are only finitely many spectral varieties $\tilde{E}_{\lambda}$.

Proof. The first statement follows from the theorem. Birational equivalence requires an isomorphism only on a Zariski open subset [Hart, p. 26]. The subset $\tilde{E}_{\lambda *}$ is open in $\tilde{E}_{\lambda}$.

Corollary 7. The varieties $\tilde{E}_{\lambda}$ are irreducible.

Proof. This follows from the connectedness of the covering spaces $\tilde{E}_{\lambda *}$ which is apparent as they are quotients of the connected space $G / H \times g_{*}$. See [GR, p. 116].

\section{Stabilizers of Weights (Parabolic Subgroups of $\boldsymbol{W}$ )}

Each spectral variety $\tilde{E}_{\lambda *}$ is isomorphic to $G / H \times \mathfrak{g}_{*} / S_{\lambda}$ for some weight $\lambda$. By Theorem 4, two spectral varieties $G / H \times \mathfrak{g}_{*} / S_{\lambda}$ and $G / H \times g_{*} / S_{\gamma}$ are isomorphic as covers of $g_{*}$ precisely when the stabilizers $S_{\lambda}$ and $S_{\gamma}$ are conjugate subgroups of $W$. In order to count the spectral varieties, a count of the conjugacy classes of stabilizers of weights follows. The algebras $E_{6}, E_{7}$, and $E_{8}$ are omitted.

Let $\alpha_{1}, \alpha_{2}, \ldots, \alpha_{n}$ be a basis of simple roots for $\mathfrak{g}$, and $\omega_{1}, \omega_{2}, \ldots, \omega_{n}$ be the dual basis of weights. Any weight $\lambda$ may then be expressed as $\lambda=\sum_{i=1}^{n} \lambda_{i} \omega_{i}$, where $\lambda_{i}=\left(\alpha_{i}, \lambda\right)$. Let $r_{j} \in W$ be the reflection across the hyperplane orthogonal to $\alpha_{j}$. The following standard results may be found in [BMP]. 


\section{Lemma 8.}

1. If two weights lie in the same orbit of $W$, then their stabilizers are conjugate in $W$.

2. The $W$ orbit of a weight contains precisely one weight in the closed Weyl chamber $\left\{\lambda=\sum \lambda_{i} \omega_{i} \mid \lambda_{i} \geqq 0\right.$ for $\left.i=1, \ldots, n\right\}$.

3. If $\lambda=\sum_{i=1}^{n} \lambda_{i} \omega_{i}$ and $\lambda_{i} \geqq 0$ for $i=1, \ldots, n$, its stabilizer $S_{\lambda}$ is generated by the set of reflections $\left\{r_{j} \mid \lambda_{j}=0\right\}$.

4. The subgroup generated by $\left\{r_{j} \mid j \in J\right\}$ for $J \subset\{1, \ldots, n\}$ is the Weyl group for the full subdiagram of the Dynkin diagram for $g$ obtained by deleting the nodes for $j \notin J$.

The lemma shows that each conjugacy class may be represented by one or more full subdiagrams of the Dynkin diagram of $\mathfrak{g}$. This gives an upper bound of $2^{n}$ conjugacy classes for an algebra of rank $n$.

The next step is to see when two subdiagrams represent the same conjugacy class. When the simple roots for $g$ are of two different lengths, this is indicated by marking (blackening) the nodes of the Dynkin diagram corresponding to the short roots. This occurs for algebras of type $B, C, F_{4}$, and $G_{2}$. Two full subdiagrams of the Dynkin diagram shall be considered isomorphic if there is a graph isomorphism between them which respects the marking of the nodes.

Proposition 9. If $\mathfrak{g}$ is a simple Lie algebra of type $A, B, C, G_{2}$, or $F_{4}$ then the set of conjugacy classes of stabilizers of weights is in one-to-one correspondence with the isomorphism classes of full subdiagrams of the Dynkin diagram for $g$ (where the isomorphisms respect the length of the roots corresponding to nodes in the diagram).

Proof. In each case the weight lattice is conveniently viewed as lying in a Euclidean space equipped with an orthonormal basis $\left\{e_{i}\right\}$ such that the Weyl group includes the permutation group on the basis elements. Dynkin's convention for numbering the nodes (simple roots) will be used [BMP].

type $A_{n}$. The weight lattice is generated by the sublattice of $\mathbf{Z}^{n+1}$ where the coordinates sum to zero and the lattice point $\left(1, \frac{-1}{n+1}, \ldots, \frac{-1}{n+1}\right)$. The Weyl group acts as the symmetric group $\Sigma_{n+1}$ on the basis vectors. The reflection $r_{j}$ corresponding to node $j$ is the permutation $(j, j+1)$.

A full subdiagram of the Dynkin diagram yields a partition as follows. Imagine the Dynkin diagram for type $A_{n}$ to have two extra edges, one extending off each end. A subdiagram is obtained by removing some of the nodes. View the missing nodes as dividers, as pictured for $n=6$ with nodes 1,3 , and 4 missing.

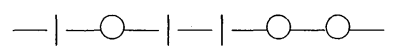

The partition of $n+1$ obtained from this subdiagram has as parts the number of edges in connected components of the picture. In the example $n+1=7=1+2+1+3$. The full subdiagram corresponds to the subgroup $\Sigma_{2} \times \Sigma_{3} \approx \Sigma_{1} \times \Sigma_{2} \times \Sigma_{1} \times \Sigma_{3} \subset \Sigma_{7}$. The $\Sigma_{2}$ associated to the second and third edges permutes $e_{2}$ and $e_{3}$, while the $\Sigma_{3}$ associated to the last three edges permutes $e_{5}, e_{6}$, and $e_{7}$. In general isomorphism classes of subdiagrams of the Dynkin diagram of type $A_{n}$ correspond one-to-one with partitions $n+1=p_{1}+\cdots+p_{k}$ which in turn correspond one-to-one with conjugacy classes of stabilizer subgroups taking the form $\Sigma_{p_{1}} \times \cdots \times \Sigma_{p_{k}}$. 
type $B_{n}$. The weight lattice is spanned by $\mathbf{Z}^{n}$ and $\left(\frac{1}{2}, \frac{1}{2}, \ldots, \frac{1}{2}\right)$. The Weyl group acts as the semidirect product $\mathbf{Z}_{2}^{n} \succ \triangleleft \Sigma_{n}$ by permuting the basis vectors and switching an arbitrary number of their signs. The reflections associated to the nodes are the permutations $r_{j}=(j, j+1)$ for $1 \leqq j \leqq n-1$ and $r_{n}=(-1)_{n}$, switching the sign on $e_{n}$. Associate to each full subdiagram a partition $n=p_{1}+\cdots+p_{k}$ by ignoring node $n$ (corresponding to the short root) and treating what remains as a subdiagram of the $A_{n-1}$ Dynkin diagram. Here $p_{k}$ is the number of edges in the component adjacent to the double bond connecting node $n$. Then if node $n$ is not part of the given subdiagram, the subdiagram represents a conjugacy class of stabilizers isomorphic to $\Sigma_{p_{1}} \times \cdots \times \Sigma_{p_{k}}$, while if node $n$ was included, the subdiagram represents a conjugacy class of stabilizers isomorphic to $\Sigma_{p_{1}} \times \cdots \times \Sigma_{p_{k-1}} \times\left(\mathbf{Z}_{2}^{p_{k}} \triangleleft \Sigma_{p_{k}}\right)$. Since the sign switch $r_{n}=(-1)_{n}$ is not conjugate to any permutation in $\Sigma_{n}$, all these conjugacy classes are distinct.

type $C_{n}$. The weight lattice $\mathbf{Z}^{n}$ with Weyl group $\mathbf{Z}_{2}^{n} \triangleleft \triangleleft \Sigma_{n}$ again acting as permutations and arbitrary sign changes. The situation is the same as in the $B_{n}$ case.

type $G_{2}$. The weight lattice consists of the sublattice of $\mathbf{Z}^{3}$ perpendicular to $(1,1,1)$. The Weyl group is the direct product $W=\mathbf{Z}_{2} \times \Sigma_{3}$ which acts by permuting the basis vectors and multiplying them all simultaneously by \pm 1 . The conjugacy classes of stabilizers are $\{e\}, W$, the class of $\mathbf{Z}_{2}$ 's inside the $\Sigma_{3}$, and the class of $\mathbf{Z}_{2}$ 's involving a permutation and multiplication by -1 . These correspond to the full subdiagrams of the Dynkin diagram. The class of permutation only $\mathbf{Z}_{2}$ 's corresponds to the single node associated to the shorter root.

type $F_{4}$. The weight lattice is generated by $\mathbf{Z}^{4}$ and $\left(\frac{1}{2}, \frac{1}{2}, \frac{1}{2}, \frac{1}{2}\right)$ and is the same as that for $D_{4}$. The Weyl group is the full automorphism group of the $D_{4}$ root system $\Sigma_{3} \times\left(\mathbf{Z}_{2}^{3}>\triangleleft \Sigma_{4}\right)$. The $\Sigma_{3}$ acts as the outer automorphisms. A hand check gives the following 12 conjugacy classes of stabilizers: $W,\{e\}, \mathbf{Z}_{2}$ (one short root), $\mathbf{Z}_{2}$ (one long root), $\mathbf{Z}_{2} \times \mathbf{Z}_{2}, \mathbf{Z}_{2}^{2} \triangleleft \triangleleft \Sigma_{2}, \Sigma_{3}$ (short), $\Sigma_{3}$ (long), $\mathbf{Z}_{2}^{4} \triangleleft \triangleleft \Sigma_{4}$ (type $B$ ), $\mathbf{Z}_{2}^{4} \triangleleft \triangleleft \Sigma_{4}$ (type C), $\Sigma_{3} \times \Sigma_{2}$, and $\Sigma_{2} \times \Sigma_{3}$, where $\Sigma_{3}$ and $\Sigma_{2}$ are associated to long and short roots in one case and vice versa in the other. The $\Sigma_{3}$ associated to the short roots may be regarded as coming from the outer automorphisms of the $D_{4}$ root system.

It turns out that two stabilizer subgroups may be abstractly isomorphic but not conjugate in $W$. These subgroups are all Weyl groups of subdiagrams of the Dynkin diagram and their structure only depends on the Coxeter graph, i.e. the Dynkin diagram without markings for root lengths. For the algebras of Proposition 9 conjugacy was determined by the isomorphism class of the marked diagram. For algebras of type $D_{n}$ this is no longer true.

In order to correlate subdiagrams with conjugacy classes of stabilizers for type $D_{n}$, recall how subdiagrams for types $A_{n-1}$ or type $B_{n}$ yield partitions of $n$. The description of conjugacy classes of stabilizer subgroups for type $D_{n}$ also uses an associated partition.

Proposition 10. Let $\mathfrak{g}$ be a simple Lie algebra of type $D_{n}$. Let $T$ map the Dynkin diagram of type $D_{n}$ to the Dynkin diagram of type $A_{n-1}$ by identifying the two nodes and their adjacent edges where the $D_{n}$ diagram forks. For each full subdiagram $D$ of the $D_{n}$ diagram, let $\wp$ be the partition $n-1=p_{1}+\cdots+p_{k}$ obtained from $T(D)$, where $p_{k}$ counts the edges in the component attached to the node obtained by identification. Let $00,01,10,11$ be a code indicating whether $D$ includes nodes $n-1$ 
and $n$ of the $D_{n}$ diagram, e.g. 10 means node $n-1$ included, node $n$ deleted. The conjugacy classes of stabilizer subgroups of $W$ correspond with the following types of data:

a) The empty graph corresponds to the (conjugacy class of the) subgroup $\{e\}$.

b) $\left(\wp, p_{k}, 11\right)$ corresponds to the conjugacy class of subgroups isomorphic to $\Sigma_{p_{1}} \times \cdots \times \Sigma_{p_{k-1}} \times\left(\mathbf{Z}_{2}^{p_{k-1}} \triangleleft \triangleleft \Sigma_{p_{k}}\right)$.

c) $(\wp, 10)$ and $(\wp, 01)$ correspond to at most two conjugacy classes of subgroups isomorphic to $\Sigma_{p_{1}} \times \cdots \times \Sigma_{p_{k}}$. The two conjugacy classes are distinct if and only if $\wp$ is an even partition.

Observe in cases b) and c) that $p_{k}>1$, while case a) covers the partition whose only parts are 1's.

Proof.

type $D_{n}(n \geqq 3)$. The weight lattice is generated by $\mathbf{Z}^{n}$ and $\left(\frac{1}{2}, \frac{1}{2}, \ldots, \frac{1}{2}\right)$. The Weyl group acts as the semidirect product $\mathbf{Z}_{2}^{n-1} \rtimes \Sigma_{n}$ by permuting the basis vectors and switching an even number of their signs. The reflections $r_{j}$ are the permutations $r_{j}=(j, j+1)$ for $1 \leqq j \leqq n-1$ and $r_{n}=(n-1, n)(-1)_{n-1}(-1)_{n}$ which switches $e_{n-1}$ and $e_{n}$ and multiplies both by -1 .

The Weyl group for $D_{n}$ may be regarded as a normal subgroup of index 2 contained in $\mathbf{Z}_{2}^{n} \triangleleft \triangleleft \Sigma_{n}$, the Weyl group for $B_{n}$. The stabilizers of weights for the type $D_{n}$ are thus intersections of stabilizers for type $B_{n}$ with $\mathbf{Z}_{2}^{n-1} \succ \triangleleft \Sigma_{n}$. Each conjugacy class for type $B_{n}$ yields either one or two conjugacy classes of type $D_{n}$. The partition associated to a subdiagram amounts to partitioning the coordinates of a weight according to equality up to sign. If an even number of sign changes can be made so that the coordinates in each equivalence class are all equal, then the conjugacy class of the stabilizer has the form $\Sigma_{p_{1}} \times \cdots \times \Sigma_{p_{k}}$ or $\Sigma_{p_{1}} \times \cdots \times \Sigma_{p_{k-1}} \times\left(\mathbf{Z}_{2}^{p_{k}} \triangleleft \Sigma_{p_{k}}\right)$ as in type $B$. When the partition $n=p_{1}+\cdots+p_{n}$ has only even terms, it is not in general possible to align the signs in the equivalence classes by an even number of sign changes. Using $(-1)_{n}$ and the Weyl group of type $D_{n}$ the signs of the coordinates in the equivalence classes can be aligned. In this case the stabilizer of the weight is, up to conjugacy, $(-1)_{n}\left(\Sigma_{p_{1}} \times \cdots \times \Sigma_{p_{k}}\right)(-1)_{n}$ which is abstractly isomorphic but not conjugate to $\Sigma_{p_{1}} \times \cdots \times \Sigma_{p_{k}} \subset \Sigma_{n} \subset \mathbf{Z}_{2}^{n-1}>\triangleleft \Sigma_{n}=$ $W$. In this case isomorphic subdiagrams do not always give conjugate stabilizers; the choice of a branch in the fork of the $D_{n}$ diagram matters. Moreover the subdiagram of type $A_{3}$ containing both branches corresponds to $\mathbf{Z}_{2}^{2} \triangleleft \Sigma_{3}$ which is abstractly isomorphic but not conjugate of $\Sigma_{4}$.

The characterizations of the conjugacy classes of stabilizers tell how many there are.

Corollary 11. Let $P(n)$ be the set of partitions of $n$ and $p(n)$ its cardinality. The number of conjugacy classes of stabilizer subgroups of the Weyl group for the simple Lie algebras of types $A, B, C, D, G_{2}$, and $F_{4}$ are as follows:

1. $A_{n} \quad p(n+1)$,

2. $B_{n}$ or $C_{n} p(n)+\sum_{P \in P(n)}(\#$ of distinct parts of $P)$,

3. $D_{n} \quad \sum_{P \in P(n)}(\#$ of distinct parts $\neq 1$ of $P)+p(n)+(\#$ of even partitions

4. $G_{2} \quad 4$,

5. $F_{4} \quad 12$. 


\section{Spectral Curves}

Many completely integrable systems can be written in the form

$$
\frac{d A}{d t}(s, t)=[A(s, t), B(s, t)]
$$

where $A$ and $B$ are functions of time $t$ and an algebraic spectral parameter $s$ and take their values in a finite dimensional Lie algebra. In the papers of Adler and van Moerbeke [AvM1,2] are a number of examples involving a rational parameter. These include the periodic Toda lattice and its generalizations to all the simple Lie algebras. In the paper of Krichever [Kr] the $\wp$-function Calogero system is written in the Lax form with an elliptic parameter.

The flows of these equations can be viewed as follows. Let $A$ be a time dependent map from a parameter space $P$ (which is an algebraic curve in the examples) to a Lie algebra g, i.e., $A: P \times \mathbf{R} \rightarrow \mathfrak{g}$. $A$ should be thought of as the solution to $(* *)$. For a representation $\rho$, let $X_{(\rho, t)}$ be the curve $X_{(\rho, t)}=\{(p, z) \in$ $P \times \mathbf{C} \mid \operatorname{det}(\rho A(p, t)-z)=0\}$ and $\tilde{X}_{(\rho, t)}$ the desingularized curve. These curves are independent of time $t$ if they arise from a Lax equation and are hence determined by their conditions, $A(-, 0): P \rightarrow \mathrm{g}$.

$$
\begin{aligned}
& \tilde{X} \\
& \downarrow \\
& P \rightarrow \mathfrak{g} \stackrel{\rho}{\rightarrow} \text { matrices }
\end{aligned}
$$

Let $\tilde{X}$ be an irreducible component of $\tilde{X}_{(\rho, t)}$. Such a curve is called a spectral curve. A flow is then obtained in the space of line bundles over the spectral curve $\tilde{X}$. This flow is obtained by observing the evolution of the eigenvector line bundle over $X$ as a function of time. Although $X$ depends on the representation $\rho$, of which there are countably many, there are only finitely many spectral curves associated to $A$ (Corollary 14).

Lemma 12. Suppose $P$ is an irreducible algebraic curve and $A: P \rightarrow \mathfrak{g}$ is an algebraic initial condition with $\operatorname{Im}(A) \cap \mathfrak{g}_{*} \neq \emptyset$. Let $P_{*}=A^{-1}\left(\mathfrak{g}_{*}\right)$, then

1. $P_{*}=P$-finite set of points.

2. The spectral curves are irreducible components of the pullbacks of

$$
\begin{array}{r}
\tilde{E}_{\lambda} \\
\downarrow \\
P_{A(-, t)}^{\longrightarrow} \mathfrak{g}
\end{array}
$$

Proof. The image of $P$ in $g$ is an irreducible algebraic curve and since the singular set is also algebraic, $\operatorname{Im}(A) \cap$ singular set is algebraic. If $\operatorname{Im}(A) \cap$ singular set is a curve then it is all of $\operatorname{Im}(A)$ but that would contradict the condition $\operatorname{Im}(A) \cap \mathfrak{g}_{*} \neq \emptyset$. Therefore $\operatorname{Im}(A) \cap$ singular set is a finite set of points. So $P_{*}$ is $P$ minus a finite set. Recall that $\mathscr{E}_{\rho}=\{(x, z) \mid \operatorname{det}(\rho((x)-z)=0\}$. The variety $X_{(\rho, t)}$ is precisely the pullback of $\mathscr{E}_{\rho}$ along $A(-, t)$,

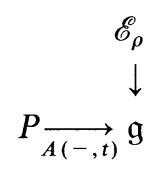


The variety $\mathscr{E}_{\rho}$ decomposes into the irreducible varieties $E_{\lambda}$ by Proposition 1 and Corollary 7. The pullbacks of

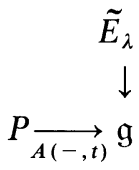

are algebraic curves $\tilde{Y}_{\lambda}$ which comprise $\tilde{X}_{(\rho, t)}$. Each spectral curve $X$ is an irreducible component of $\tilde{X}_{(\rho, t)}$ for some $\rho$ and so of $\tilde{Y}_{\lambda}$.

Suppose $P$ is as in Lemma 12 . Let $B W$ be the classifying space for principal $W$-bundles and let $E W$ be the universal cover of $B W$. Principal $W$-bundles over a space $T$ (including regular $W$-covering spaces) are given as pullbacks of $E W$ along maps $T \rightarrow B W$, bundles corresponding to homotopy classes of maps. Since $G / H \times \mathfrak{h}_{*} \rightarrow \mathfrak{g}_{*}$ is a regular $W$-cover it can be given as $\varepsilon^{*}(E W)=G / H \times \mathfrak{h}_{*}$ for some map $\varepsilon: \mathfrak{g}_{*} \rightarrow B W$. The following theorem gives the classification of spectral curves.

Theorem 13. Suppose $P$ is an irreducible algebraic curve and $A: P \rightarrow \mathfrak{g}$ is an algebraic map with $\operatorname{Im}(A) \cap \mathfrak{g}_{*} \neq \emptyset$. Let $M$ be the image of $\pi_{1}\left(P_{*}\right)$ under $(\varepsilon \circ A)_{*}$ : $\pi_{1}\left(P_{*}\right) \rightarrow W$. If $\lambda$ is a weight let $S_{\lambda} \subset W$ be its stablizer and $M_{\lambda}=S_{\lambda} \cap M$. Then

(1) There is a one to one correspondence between spectral curves with their projections, $X \rightarrow P$, and conjugacy classes of the subgroups $M_{\lambda}$ in $M$.

(2) If $X_{M} \rightarrow P$ is the spectral curve corresponding to the trivial subgroup of $M$ then the others arise as quotients $X_{M} / M_{\lambda} \rightarrow P$.

Proof. Let $\hat{Y}_{*}$ be the pullback $A^{*}\left(G / H \times \mathfrak{h}_{*}\right)$ so that $\tilde{Y}_{*} \rightarrow P_{*}$ is a principal $W$ bundle. Let $e \in \tilde{Y}_{*}$ so $W \cdot e$ is a fiber and let $\tilde{X}_{*}$ be the component of $\tilde{Y}_{*}$ containing $e$. Since $\operatorname{Im}(\varepsilon \circ A)_{*}=M, \underset{\tilde{X}_{*}}{\underset{\tilde{Y}}{P_{*}}} P_{*}$ is a regular $M$-cover. Since the action of $W$ is transitive on any fiber of $\tilde{Y}_{*}^{*}, \tilde{Y}_{*}$ is isomorphic as a variety to $|W / M|$ copies of $\tilde{X}_{*}$.

Suppose $w_{1} \cdot e$ and $w_{2} \cdot e$ are in the same fiber. They are in the same component if and only if $w_{2}^{-1} w_{1} \in M$, i.e., $w_{1}$ and $w_{2}$ represent the same left coset in $W / M$. The subgroup of $W$ that preserves the component $w \cdot \tilde{X}_{*}$ is $w M w^{-1}$. By Lemma 12 and Theorem 4, the spectral curves arise from components of $\hat{Y}_{*} / S_{\lambda}$. The components of $\tilde{Y}_{*} / S_{\lambda}$ correspond to the double cosets $S_{\lambda} \backslash W / M$. Let $X$ be a component and take a $w \in W$ such that the image of $w \cdot e$ under $\tilde{Y}_{*} \rightarrow \tilde{Y}_{*} / S_{\lambda}$ is in $X$. The curve $X$ is $w \cdot \tilde{X}_{*} /\left(w M w^{-1} \cap S_{\lambda}\right)$. But $w \cdot \tilde{X}_{*} /\left(w M w^{-1} \cap S_{\lambda}\right) \cong \tilde{X}_{*} / M \cap w^{-1} S_{\lambda} w=\tilde{X}_{*} / M \cap S_{\gamma}$ $=\tilde{X}_{*} / M_{\gamma}$ for $\gamma=w^{-1} \cdot \lambda$. Parts (1) and (2) follow.

Corollary 14. If $P$ is a closed irreducible algebraic curve and $A: P \rightarrow g$ is an algebraic initial condition with $\operatorname{Im}(A) \cap \mathfrak{g}_{*} \neq \emptyset$ then $A$ has only finitely many spectral curves.

Suppose $\tilde{\tilde{Y}} \stackrel{\rho_{1}}{\rightarrow} \tilde{Y} \stackrel{\rho_{2}}{\rightarrow} Y$ is a sequence of algebraic covers with $\tilde{\tilde{Y}} \rightarrow Y$ a regular $G$-cover. There is then a subgroup $H \subset G$ with $\tilde{\tilde{Y}} \rightarrow \tilde{Y}$ a regular $H$-cover. Let $F_{\tilde{Y}}$, $F_{\tilde{Y}}$ and $F_{Y}$ denote the field of meromorphic functions on the given variety. These can all be considered as subfields of $F_{\tilde{Y}}$ via $\rho_{1}$ and $\rho_{2} \circ \rho_{1}$. It is then a standard result ([F] page 57) that $\operatorname{Gal}\left(F_{\tilde{Y}}, F_{Y}\right)=G$ and $\operatorname{Gal}\left(F_{\tilde{Y}}, F_{\tilde{Y}}\right)=H$. If $H$ is normal in $G$ then $\operatorname{Gal}\left(F_{\tilde{Y}}, F_{Y}\right)=G / H$.

Theorem 15. Suppose $X_{M} \rightarrow P$ is the spectral curve corresponding to the trivial subgroup of $M$ and $F_{P} \rightarrow F_{X_{M}}$ is the induced embedding of the function fields. Then the 
Galois group $\operatorname{Gal}\left(F_{X_{M}}, F_{P}\right)$ is $M$ and the subfield fixed by $M_{\lambda}$ is the function field of the spectral curve $X_{M} / M_{\lambda} \rightarrow P$.

This theorem sets up a one to one correspondence between spectral curves and fixed fields arising from stabilizers. It is just a reinterpretation of Theorem 13.

Proposition 16. Let $q_{M, \lambda}(s, z)=\prod_{\gamma \in M \cdot \lambda}\left(\gamma\left(\operatorname{Ad}_{g}\left(A(s)_{\text {semi-simple }}\right)\right)-z\right)$ for $(s, z)$ $\in P \times \mathbf{C}$. Then $q_{M, \lambda}$ is irreduicble and the normalization of the curve defined by $q_{M, \lambda}(s, z)=0$ is isomorphic to $X_{M} / M_{\lambda}$.

Proof. Recall from Proposition 1 that $p_{\lambda}(x, z)=\prod_{\gamma \in W \cdot \lambda}\left(\gamma\left(\operatorname{Ad}_{g}\left(x_{\text {semi-simple }}\right)\right)-z\right)$, where $x \in \mathfrak{g}$. The variety $E_{\lambda}$ is defined by $p_{\lambda}(x, z)=0$. By the construction of the pullback in Lemma 12, $\tilde{Y}_{\lambda *}=\left\{(s, x, z) \in P \times \mathfrak{g} \times \mathbf{C} \mid A(s)=x\right.$ and $\left.P_{\lambda}(x, z)=0\right\}$. The polynomial $p_{\lambda}(A(s), z)$ factors as

$$
\left.p_{\lambda}(A(s), z)=\prod_{c \in M \backslash W / S_{\lambda}} \prod_{m \in M / M_{c \cdot i}} m c \lambda\left(\operatorname{Ad}_{g}\left(A(s)_{\text {semi-simple }}\right)\right)-z\right),
$$

where $c$ and $m$ each run through a set of coset representatives. The factorization may be rewritten as $p_{\lambda}(A(s), z)=\prod_{c \in M \backslash W / S_{i}} q_{M, c \cdot \lambda}(A(s), z)$. The factorization is a rephrasing of the decomposition of $Y_{\lambda *}=\tilde{Y}_{*} / S_{\lambda}$ into connected components as discussed in the proof of Theorem 13. As each $q_{M, c \cdot \lambda}$ defines a connected curve, it is irreducible. By the proof of Theorem 13, the desingularization of the curve defined by $q_{M, c \cdot \lambda}=0$ is $X_{M} / M_{c \cdot \lambda}$.

In the special cases $M=W$ or $S_{\lambda}=\{e\}$, the curves defined by $q_{M, c \cdot \lambda}$ are isomorphic to each other, i.e. they don't depend on $c$.

Proposition 17. The splitting field of $p_{\lambda}(A(s), z)$ for $\lambda \neq 0$ is independent of $\lambda$ up to isomorphism of field extensions of $F_{P}$.

Proof. Let $\lambda$ and $\gamma$ be two non-zero weights of $g$. Let $s_{0}$ be a value in the parameter space $P$ such that $p_{\lambda}\left(s_{0}, z\right)$ and $p_{\gamma}\left(s_{0}, z\right)$ have only simple roots in $z$. These roots are meromorphic functions of $s$ in a neighborhood of $s_{0}$. The polynomials $p_{\lambda}$ and $p_{\gamma}$ with coefficients in the function field $F_{P}$ split in the field of germs of meromorphic functions at $s_{0}$. In particular the splitting field of $p_{\lambda}$ is $F_{P}\left(\left\{r_{w \cdot \lambda} \mid w \in W / S_{\lambda}\right\}\right)$, where $r_{w \cdot \lambda}$ is the germ of the function $w \cdot \lambda\left(\operatorname{Ad}_{g(s)}(A(s))_{\text {semi-simple }}\right)$, appearing in the formula for $p_{\lambda}$ in Proposition 1. Here $g(s) \in G$ is independent of $\lambda$. The weights $w \cdot \lambda$ span $\mathfrak{h}^{*}$ and in fact $\gamma$ is a rational linear combination of $\{w \cdot \lambda\}$. Hence the germs $r_{w \cdot \gamma}$ which generate the splitting field $F_{P}\left(\left\{r_{w \cdot \gamma}\right\}\right)$ lie in the field $F_{P}\left(\left\{r_{w \cdot r}\right\}\right)$. The argument is symmetric in $\lambda$ and $\gamma$ so the two splitting fields are equal.

If $(\varepsilon \circ A)_{*}$ is onto $W$ (i.e., $\left.M=W\right)$ then the spectral curves correspond to the conjugacy classes of the stabilizers of weights, $S_{\lambda}$. This is the expected situation (it is generic for the examples) although not the exclusive situation. For example, if $\operatorname{Im}(A) \subset \mathfrak{h}$ then $X=P$ is the only spectral curve. Now consider the case $M=W$. Here the curves $X_{\lambda}=X_{M} / M_{\lambda}$ are defined by $p_{\lambda}(A(s), z)$. The function field $F_{X_{\dot{\lambda}}}$ is isomorphic to $\mathbf{C}(s, z) /\left(p_{\lambda}(A(s), z)\right)$.

Corollary 18. Suppose $M=W$.

1. $p_{\lambda}(A(s), z)$ is irreducible over $F_{P}$.

2. If $S_{\lambda}$ is a normal subgroup of $W$ then $S_{\lambda}=\{e\}$ or $W$. 
Proof. The first statement follows from $p_{\lambda}(A(s), z)=q_{W, \lambda}(s, z)$.

If $\lambda \neq 0$ and $S_{\lambda}$ is normal in $W$ then $X_{\lambda *} \rightarrow P_{*}$ is regular (i.e. $F_{P} \subset F_{X_{1}}$ is Galois) and so $F_{X_{\lambda}}$ is the splitting field of $p_{\lambda}(A(s), z)$. By Proposition $17, F_{X_{W}}=F_{X}$, and $S_{\lambda}=\{e\}$ (Theorem 15).

The construction of the monodromy group and the criterion below will be useful for some particular examples. If $p \in P_{*}$ then $\pi_{1}\left(P_{*}\right)$ acts (on the right) on the set $\rho^{-1}(p)$. Furthermore, $\rho^{-1}(p)=\rho_{*} \pi_{1}\left(X_{*}^{*}\right) \backslash \pi_{1}\left(P_{*}\right)$ as a $\pi_{1}\left(P_{*}\right)$-module. This is the monodromy representation of $\pi_{1}\left(P_{*}\right)$. Let $\tilde{X}_{*}$ be an irreducible component of $A^{*}\left(G / H \times \mathfrak{h}_{*}\right)$. The image of $\pi_{1}\left(P_{*}\right)$ in Aut $\left(\rho^{-1}(p)\right)$ is the monodromy group. If $\tilde{X}_{*}$ is a regular cover then the monodromy group is isomorphic to the group of covering translations.

Proposition 19. Suppose $X_{M *} \rightarrow P_{*}$ is a regular cover with group of translations $M$ and that $\tilde{X}_{*}=X_{M *} / J$ for $J \subset M$. If $N$ is the largest subgroup of $J$ which is normal in $M$ then the monodromy group for $\tilde{X}_{*} \rightarrow P_{*}$ is $M / N$.

The following is the criterion that will be used in the next section.

Criterion. If $\pi_{1}\left(P_{*}\right)$ acts by $|W|$ distinct permutations on the monodromy for any one spectral curve then $M=W$ and $(\varepsilon \circ A)_{*}$ is onto $W$.

Proof of Proposition 19. If $\alpha \in \pi_{1}\left(P_{*}\right)$ then $\alpha$ acts in the automorphisms of a fiber via path lifting. So the monodromy representation $f: \pi_{1}\left(P_{*}\right) \rightarrow \operatorname{Aut}\left(\rho^{-1}(p)\right)$ factors through $M$ since any path lifts above $\tilde{X}_{*}$ to $X_{M *}$. Let $K$ be the kernel of $f$. For an $\alpha \in \pi_{1}\left(P_{*}\right), \alpha \in K$ if and only if every lift of $\alpha$ to $\tilde{X}_{*}$ is a loop, i.e., $\beta \alpha \beta^{-1} \in \pi_{1}\left(\tilde{X}_{*}\right)$ for all $\beta \in \pi_{1}\left(P_{*}\right)$. Therefore $K$ is the largest normal subgroup of $\pi_{1}\left(P_{*}\right)$ contained in $\pi_{1}\left(\tilde{X}_{*}\right)$. The nesting of the subgroups $\pi_{1}\left(X_{M *}\right) \subset K \subset \pi_{1}\left(\tilde{X}_{*}\right) \subset \pi_{1}\left(P_{*}\right)$ yields $\pi_{1}\left(P_{*}\right) / \pi_{1}\left(X_{M *}\right)=M, \pi_{1}\left(\tilde{X}_{*}\right) / \pi_{1}\left(X_{M *}\right)=J$ and $K / \pi_{1}\left(X_{M *}\right)=N$. Therefore $M / N \cong \pi_{1}\left(P_{*}\right) / K$ which is isomorphic to the image of $f$. Note that $X_{M *} / N$ is the smallest regular cover of $P_{*}$ over $\tilde{X}_{*}$.

\section{The Toda Lattice}

This section examines a collection of specific integrable systems, the periodic Toda lattices. It is shown that for generic initial conditions the criterion developed in the previous section applies, giving a one-to-one correspondence between the spectral curves for such an initial condition and the conjugacy classes of parabolic subgroups of the Weyl group of an underlying Lie algebra. This classification is the same as that given in Theorem 4 for the eigenvalue covers of $\mathfrak{g}_{*}$ (i.e. for spectral varieties).

The generalized periodic Toda lattice is a system of Hamiltonian equations that in canonical coordinates $q_{1}, \ldots, q_{n}, p_{1}, \ldots, p_{n}$ is

$$
\frac{d q_{i}}{d t}=\frac{\partial H}{\partial p_{i}} \quad \frac{d p_{i}}{d t}=-\frac{\partial H}{\partial q_{i}} .
$$

The Hamiltonian function is given by

$$
H(q, p)=\frac{1}{2} \sum_{i=1}^{n} p_{i}^{2}+\sum_{\alpha \in \tilde{\Pi}} e^{2(q, \alpha)} .
$$


This Hamiltonian depends on the underlying Lie algebra $\mathfrak{g}$. The position $q=\left(q_{1}, \ldots, q_{n}\right)$ and the momentum $p=\left(p_{1}, \ldots, p_{n}\right)$ are viewed as elements of a fixed Cartan subalgebra $\mathfrak{h}$. The entries $q_{i}$ and $p_{i}$ are their coordinates in the epsilon basis (see [BMP, table 2] or [H2, pp. 64-65]). In the cases of the algebras $A_{n}$ and $G_{2}$ the Cartan subalgebra consists of those elements $\left(q_{1}, \ldots, q_{n}\right)$ such that $\sum q_{i}=0$, rather than the whole space spanned by the epsilon basis. If the equations are interpreted as the motion of a system of particles, this restriction amounts to reducing the Hamiltonian system by normalizing the center of mass to the origin. The $E_{6}$ and $E_{7}$ root systems are viewed as subsystems of the $E_{8}$ root system in the epsilon basis. The set $\tilde{\Pi}$ consists of a simple basis $\Pi$ of the roots of $g$ with respect to $\mathfrak{h}$ along with the negative of the highest root relative to this simple basis. The pairing $(q, \alpha)$ is just duality as the roots lie in $\mathfrak{h}^{*}$, the dual of $\mathfrak{h}$.

The Toda lattice, both periodic and non-periodic (replace $\tilde{\Pi}$ by $\Pi$ in the formula for $H$ ) for the algebra of type $A_{n}$ was devised by $M$. Toda to provide a tractable mathematical model of vibrations in a nonlinear lattice. His purpose was to shed some light on numerical experiments which displayed unexpected periodicity in the partition of energy among linear modes and later experiments displaying soliton behavior in nonlinear lattices (see Toda's book [T]). These Toda lattices of type $A$ have the mechanical interpretation of one dimensional lattices of particles with a directed exponential interaction between nearest neighbors. A Lax equation for the Toda lattice of type $A_{n}$ was found by Flaschka. The generalized periodic Toda lattices, i.e. for arbitrary simple Lie algebras, and their Lax equation versions were found by Bogoyavlensky [Bo], although without a spectral parameter. Their complete integrability is proven in [AvM1]. Many other people have studied these equations (see the survey article [OP]).

The periodic Toda lattice may be expressed in the Lax form $\dot{A}=[A, B]$ with a spectral parameter $s$ by taking

$$
\begin{aligned}
& A=p+\sum_{\alpha \in \Pi} e^{(q, \alpha)}\left(E_{\alpha}+E_{-\alpha}\right)+e^{(q, \Omega)}\left(s^{-1} E_{\Omega}+s E_{-\Omega}\right) \\
& B=\sum_{\alpha \in \Pi} e^{(q, \alpha)}\left(E_{\alpha}-E_{-\alpha}\right)+e^{(q, \Omega)}\left(-s^{-1} E_{\Omega}+s E_{-\Omega}\right) .
\end{aligned}
$$

Here $\Pi$ is a basis of the simple roots and $\Omega$ is the highest root, so $\tilde{\Pi}=\Pi \cup\{-\Omega\}$. The vectors $E_{\alpha}$ are the members of a Chevalley basis belonging to the root spaces corresponding to the roots $\alpha$. The position $q$ and the momentum $p$ are elements of $\mathfrak{h}$. The Lax equation works out to be equivalent to the Hamiltonian formulation since the difference of any two roots in $\tilde{\Pi}$ is not a root. In the explicit matrices given below the variable $p$ will be represented by entries $b_{1}, b_{2}, \ldots$ and the expressions $e^{(q, \alpha)}$ will be represented by the entries $a_{1}, a_{2}, \ldots$.

For each finite dimensional representation $\rho$ of the underlying Lie algebra $g$ the characteristic polynomial $\operatorname{det}(\rho(A(s))-z)$ is invariant under the flow of the equations. The spectral curves are the normalized irreducible components of the curves defined by the equations $\operatorname{det}(\rho(A(s))-z)=0$ for the various representations $\rho$.

The spectral curves depend on the initial conditions for the differential equations. The following proposition describes the family of spectral curves obtained from a fixed initial condition. The description is not valid for every initial condition, but holds for a generic set of initial conditions. The type $G_{2}$ case can, in fact, be viewed as a non-generic case for initial conditions in the algebra of type $B_{3}$. By 
a generic set of initial conditions is meant, for each algebra considered, a Zariski open subset of the initial conditions where the set of initial conditions is viewed as lying in $\mathbf{C}^{N}$ for some $N$ by means of the coordinates $\left\{a_{i}, b_{i}\right\}$ mentioned above and appearing in the explicit matrices given in the proof of the proposition.

Proposition 20. Let $\mathfrak{S}$ be the set of spectral curves for the generalized Toda lattice equations of type $A, B, C, D$, or $G_{2}$ and a fixed initial condition. Regard two spectral curves in $\subseteq$ as the same if there is an isomorphism between them commuting with their projections onto the parameter space (the Riemann sphere, $\mathbf{P}$ ). The following results hold for a generic set of initial conditions.

a) The members of $\mathfrak{\subseteq}$ are in one-to-one correspondence with the conjugacy classes of parabolic subgroups of the Weyl group belonging to the simple Lie algebra of type corresponding to the equations.

b) The members of $\mathfrak{G}$ are precisely the pullbacks $X_{\lambda}$ of the eigenvalue covers $\tilde{E}_{\lambda}$

$$
\begin{gathered}
X_{\lambda} \rightarrow \tilde{E}_{\lambda} \\
\downarrow \\
\mathbf{P} \rightarrow \mathfrak{g}
\end{gathered}
$$

where the map $\mathbf{P} \rightarrow \mathfrak{g}$ sends $s$ to $A(s)$ at $t=0$.

c) Let $\rho$ be a faithful irreducible representation of the underlying Lie algebra $\mathrm{g}$ and $A$ be a generic initial condition. Let $K$ be the splitting field of a nontrivial irreducible factor of the characteristic polynomial $\operatorname{det}(\rho(A(s))-z)$ as a polynomial in $z$ with coefficients in $\mathbf{C}(s)$. Then the Galois group $\operatorname{Gal}(K, \mathbf{C}(s))$ is isomorphic to the Weyl group for $\mathfrak{g}$.

Proof. The bulk of the proof consists of a case by case analysis proving that $(\varepsilon \circ A)_{*}: \pi_{1}\left(\mathbf{P}_{*}\right) \rightarrow W$ is an epimorphism using the criterion following Proposition 19. It then follows that $M=W$. Part a) is then a special case of Theorem 13. Part b) is the construction in Lemma 12 and Proposition 16. Part c) follows from Theorem 15 and Corollary 18 . The criterion will be applied to specific spectral curves for generic initial conditions. The specific spectral curve arises from the highest weight of the lowest dimensional faithful representation of $\mathfrak{g}$, the classical representation when $\mathfrak{g}$ is $A_{n}, B_{n}, C_{n}$ or $D_{n}$. Hence if $\lambda$ is the lowest nonzero dominant weight and $\rho$ is the classical representation then $\widetilde{\mathscr{E}}_{\rho}=\widetilde{E}_{\lambda}$ in the notation from the section spectral varieties. The spectral curves $X_{\lambda} \rightarrow \mathbf{P}$ all arise from $p_{\lambda}(A(s), z)=0$ and the parameter space $\mathbf{P}$ is the Riemann sphere. The matrices displayed are taken from [AvM1].

Now consider the case where $\mathrm{g}$ is of type $A_{n-1}$. In the classical representation of $\operatorname{sl}(n, \mathbf{C})$ the matrix $A$ in the Lax equation $\dot{A}=[A, B]$ takes the tridiagonal form

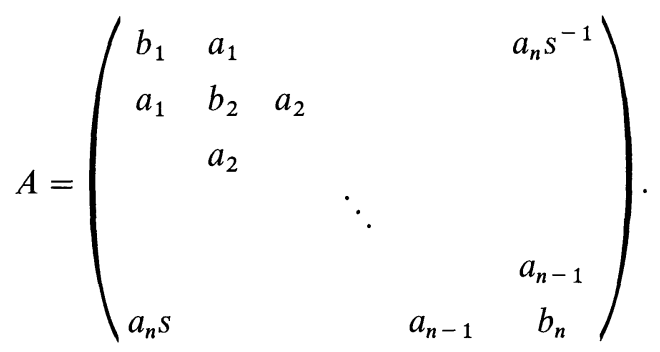


The center of mass is normalized to zero so $\sum b_{i}=0$ and $\prod a_{i}=1$.

The characteristic polynomial $p(s, z)=\operatorname{det}(A(s)-z)$ takes the form

$$
(-1)^{n}\left(z^{n}+c_{n-2} z^{n-2}+\cdots+c_{1} z+c_{0}+\tau\right),
$$

where $c_{0}, \ldots, c_{n-2}$ are complex numbers (functions of the phase variables $\left\{a_{i}, b_{i}\right\}$ which are constants of the motion) and $\tau=-\left(s+s^{-1}\right)$. Generic initial conditions imply generic coefficients $c_{i}$ in the characteristic polynomial for $A$ [vM, Theorem 2.1]. The following lemma is proven in [Mc].

Lemma. For generic coefficients $c_{0}, \ldots, c_{n-2} \in \mathbf{C}$, the Riemann surface $X$ defined $b y$ $0=p(\tau, z)=z^{n}+c_{n-2} z^{n-2}+\cdots+c_{0}+\tau$ has the following properties:

(a) The projection $h: X \rightarrow \mathbf{P}$ has branch points at $\infty$ and $n-1$ additional points in $\mathbf{P}$. Winding once around $\infty \in \mathbf{P}$ cyclically permutes the $n$ sheets of $X$ over $\mathbf{P}$. Winding once around one of the other branch points switches a pair of sheets of $X$.

(b) The image of $\pi_{1}(\mathbf{P}-$ branch points $)$ in the group of permutations of the sheets of $X$ is the full permutation group $\Sigma_{n}$. In other words, the Galois group of the splitting field of $p(\tau, z)$ over the field $\mathbf{C}(\tau)$ is $\Sigma_{n}$.

Part (b) says the monodromy group is $\Sigma_{n}$. If $\tau$ is replaced by $-\left(s+s^{-1}\right)$, the consequence is to have two copies of the branch points in the lemma, and so the monodromy group is still $\Sigma_{n}$. This gives the result for type $A_{n-1}$.

The case of the algebras of type $B_{n}$, i.e. $s o(2 n+1, \mathbf{C})$, is handled similarly. As described in [AvM1] in the classical representation the matrix $A$ in the Lax equation is given by

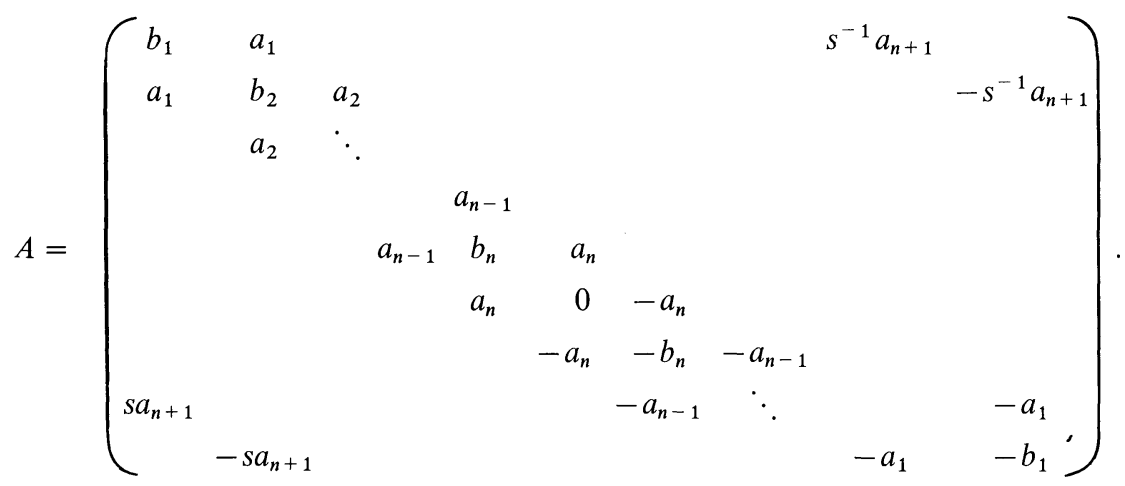

Due to the symmetry of the matrix, the characteristic polynomial is invariant under the changes $z \rightarrow-z$. Since this polynomial has odd degree, it takes the form $p(s, z)=\operatorname{det}(A-z)=z q\left(s, z^{2}\right)$. Moreover, by inspection, the spectral parameter $s$ appears only in the constant term which takes the form $c_{0}+(-1)^{n+1}\left(s+s^{-1}\right)\left(2 a_{1} b_{1} a_{2}^{2} a_{3}^{2} \ldots a_{n}^{2}\right)$. Hence the polynomial defining the spectral curve has the form

$$
q\left(s, z^{2}\right)=z^{2 n}+c_{n-1} z^{2 n-2}+\cdots+c_{1} z^{2}+c_{0}+\tau,
$$

where $\tau$ is a constant times $s+s^{-1}$. Again the constants $c_{i}$ are generic when the initial condition $A(0)$ is generic, since for a given value of $s$, the matrix may be arbitrarily close to a diagonal matrix in $\mathfrak{h}$. The roots of $q\left(z^{2}\right)$ as a polynomial over 
C(s) may be used to label the sheets of a dissection of the curve as a covering. For a fixed $s$ the roots $r_{1}, r_{2}, \ldots r_{n},-r_{1},-r_{2}, \ldots,-r_{n}$ form a fiber over $s$ on which the monodromy group acts.

The monodromy group is the image of $(\varepsilon \circ A)_{*}$. From the previous case this group maps onto $\Sigma_{n}$ by the map squaring $z$. Also winding around one of the two branch points where the constant coefficient of $q\left(z^{2}\right)$ vanishes interchanges one pair $\left\{r_{i},-r_{i}\right\}$ and leaves the rest of the sheets unchanged. These actions generate the whole group $Z_{2}^{n} \triangleleft \Sigma_{n}$, showing that it is the monodromy group of the cover.

The case of the algebras of type $C_{n}$ is almost the same as for type $B_{n}$. Again, as described in [AvM1] the matrix $A$ in the Lax equation is

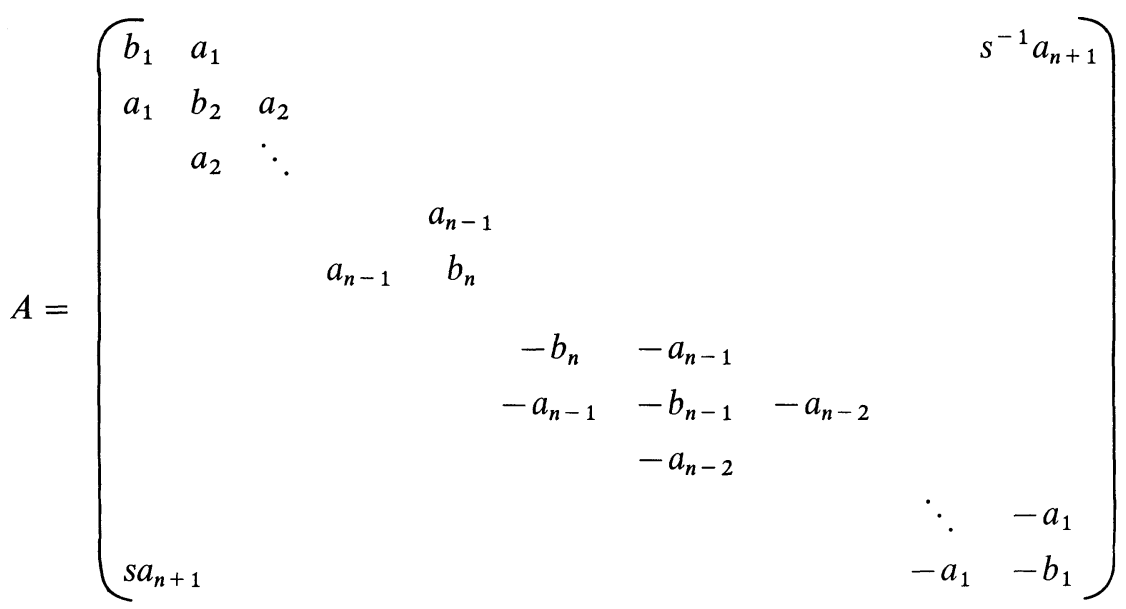

The characteristic polynomial takes the form

$$
p(s, z)=q\left(s, z^{2}\right)=z^{2 n}+c_{n-1} z^{2 n-2}+\cdots+c_{1} z^{2}+c_{0}+\tau,
$$

where $\tau=\left(s+s^{-1}\right)\left(\prod a_{i}^{2}\right)$ and the constants $c_{i}$ are generic. From here the argument proceeds as for type $B_{n}$.

For type $D_{n}$, the matrix for $A$ in the classical representation is

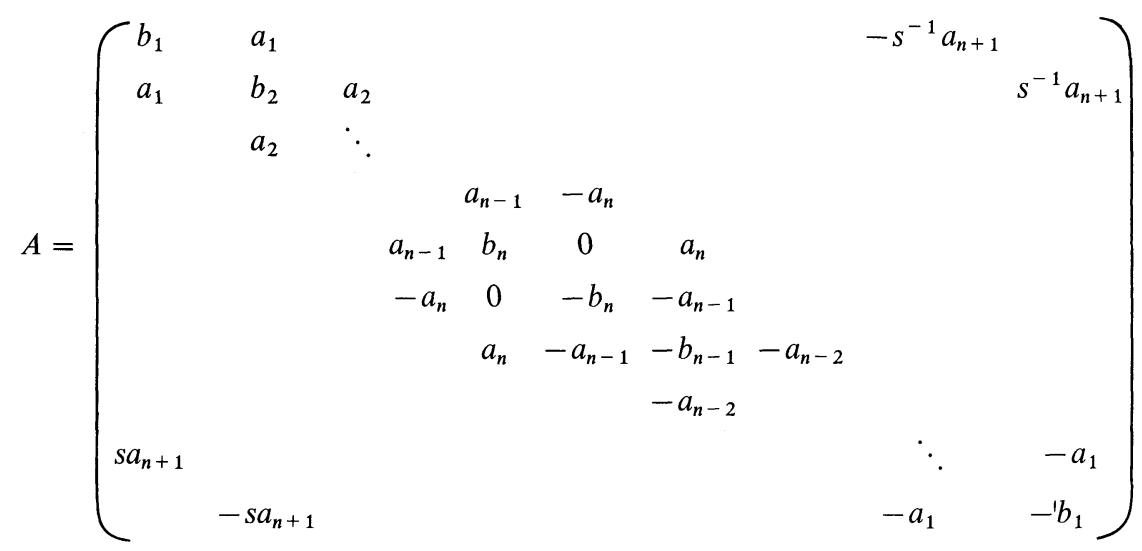


The characteristic polynomial takes the form

$$
p(s, z)=q\left(s, z^{2}\right)=z^{2 n}+c_{n-1} z^{2 n-2}+\cdots+c_{1} z^{2}+\tau z^{2}+c_{0},
$$

where $\tau=4\left(s+s^{-1}\right)(-1)^{n-1}\left(a_{2} \ldots a_{n-2}\right)^{2}\left(a_{1} a_{n-1} a_{n} a_{n+1}\right)$ and again the constants $c_{i}$ are generic.

Label the sheets of the Riemann surface defined by $p(\tau, z)=0$ as a cover of the $\tau$-sphere by $r_{1}, r_{2}, \ldots, r_{n},-r_{1},-r_{2}, \ldots,-r_{n}$. The cover has branch points above $\tau=\infty$ and above $n$ other points. Label the sheets by their signs and subscripts. Then winding once around $\infty$ induces the permutation $(1,-1)$ $(2,3, \ldots, n,-2,-3, \ldots,-n)$ for a convenient choice of labels. Winding once around any of the other $n$ branch points yields a permutation in the conjugacy class within the group $W=Z_{2}^{n-1} \triangleleft \triangleleft \Sigma_{n}$ of signed permutations of the form $(i, j)(-i,-j)$ where $i \neq \pm j$. By relabeling the sheets if necessary, one of these permutations may be taken to be $(1,2)(-1,-2)$ without changing the name for the permutation at $\infty$.

Let $G$ denote the group generated by the permutations induced by winding around the branch points, i.e. the monodromy group to be calculated. Each of the generators lies in $Z_{2}^{n-1} \triangleleft \triangleleft \Sigma_{n}$. It is sufficient to show that the two permutations $\sigma=(1,-1)(2,3, \ldots, n,-2,-3, \ldots,-n)$ and $v=(1,2)(-1,-2)$ generate $Z_{2}^{n-1} \triangleleft \triangleleft \Sigma_{n}$. First observe that the elements $\sigma^{k} v \sigma^{-k}$ generate the permutation group of the symbols $\{1,2,-3,4,-5, \ldots,-n\}$ for $n$ odd, and at least this group for $n$ even. The group $Z_{2}^{n-1}>\triangleleft \Sigma_{n}$ is contained in an exact sequence

$$
\{e\} \rightarrow Z_{2}^{n-1} \rightarrow Z_{2}^{n-1} \rtimes \Sigma_{n} \rightarrow \Sigma_{n} \rightarrow\{e\}
$$

which contains the exact sequence of groups

$$
\{e\} \rightarrow \operatorname{Ker} \rightarrow G \rightarrow \Sigma_{n} \rightarrow\{e\} .
$$

The map from $G$ to $\Sigma_{n}$ is onto, so it suffices to show that the kernel Ker is all of $Z_{2}^{n-1}$. As a $\Sigma_{n}$ module $Z_{2}^{n-1}$ is generated by any element other than \pm the identity. If $n$ is odd then $\sigma^{n-1}$ acts as $(-1,1,1, \ldots, 1)$ and represents a nontrivial member of Ker. If $n$ is even, then $\sigma$ itself is not in the permutation group of $\{1,2,-3,4,-5, \ldots\}$ and so represents a nontrivial element of Ker. This shows that $G=Z_{2}^{n-1} \triangleleft \Sigma_{n}$, the Weyl group for type $D_{n}$. This clarifies the discussion in [AvM, p. 281] where it is incorrectly stated that generic coefficients can be treated as indeterminates giving the impression that the $B_{n}$ and $D_{n}$ cases would have the same Galois group.

Lastly, the case of $G_{2}$ is interesting as it can be viewed as an example of non-generic initial conditions for $B_{3}$. If the initial condition were viewed as a map into $B_{3}$ then $M$ does not equal the Weyl group of $B_{3}$. However as a map into $G_{2}$, $M$ is the Weyl group of $G_{2}$. After reversing time and exchanging $s$ and $s^{-1}$, the matrix for $A$ is realized in [AvM1] as the following special case of the type $B_{3}$ matrix.

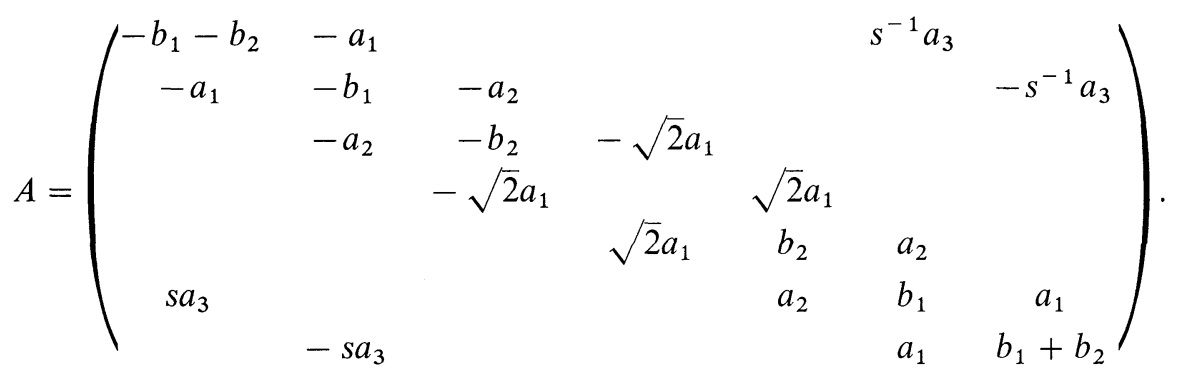


The characteristic polynomial of this matrix, after dividing by $z$, has the form $p(s, z)=z^{2}\left(z^{2}-c_{1}\right)^{2}+c_{0}+\tau$, where $\tau$ is a constant times $s+s^{-1}$. The constant $c_{1}$ is $a_{2}^{2}+3 a_{1}^{2}+b_{2}^{2}+a_{3}^{2}+b_{1}^{2}+b_{1} b_{2}$. The other constants are rather, long expressions.

The curve defined by the vanishing of the characteristic polynomial forms a Riemann surface of six sheets covering the $\tau$-sphere. The sheets may be designated $r_{1}, r_{2}, r_{3},-r_{1},-r_{2},-r_{3}$, where $r_{1}+r_{2}+r_{3}=0$ as functions of $\tau$. There are three branch points $\infty, 0$, and $\tau=-c_{0}-4 / 27 c_{1}^{3}$. Winding once about $\infty$ induces the permutation $(1,-2,3,-1,2,-3)$. Again the $r$ 's are suppressed in the notation. Winding once about 0 induces the permutation $(1,-1)(2,-2)(3,-3)$. This differs from the generic situation for type $B_{3}$. Finally winding about the remaining branch point yields the permutation $(1,2),(-1,-2)$ for a suitable labelling of the sheets. This last winding cannot interchange, say, 1 and -2 because it must preserve the relationship that $r_{1}+r_{2}+r_{3}=0$. These three permutations generate the dihedral group of order 12, i.e. the Weyl group for $G_{2}$.

The collapse in symmetry from type $B_{3}$ to type $G_{2}$ may be viewed geometrically. The Weyl group for type $B_{3}$ is the symmetry group of the octahedron. When the axes of the octahedron are pushed into a coplanar position (because the roots of the characteristic polynomial become linearly dependent), the symmetry group is reduced to that of a hexagon, which is the Weyl group for type $G_{2}$.

The paper concludes with a description of the spectral curves for the periodic Toda lattice of type $A_{3}$, i.e. $\operatorname{sl}(4, \mathbf{C})$ or equivalently $\operatorname{so}(6, \mathbf{C})$. In [Mc] two of the spectral curves for the Toda lattice of type $A_{3}$ are discussed in detail. A correspondence is exhibited between them and the mappings it induces on homology and on holomorphic differentials are given very explicitly. The entire family of spectral curves and their coverings of one another forms a lattice as pictured.

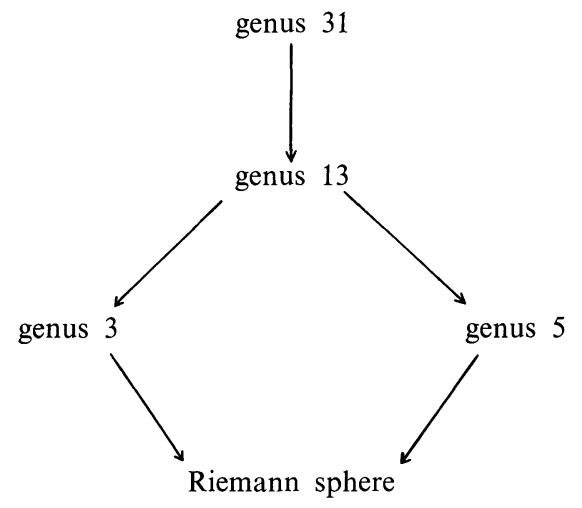

These curves may be labelled by either Dynkin diagrams, partitions of 4 , or subgroups of the Weyl group $\Sigma_{4}$. Here is a table.

\begin{tabular}{|c|c|c|c|}
\hline genus & diagram & partition & subgroup of $\Sigma_{4}$ \\
\hline 31 & empty & $1+1+1+1$ & $\{e\}$ \\
\hline 13 & 0 & $1+1+2$ & $\Sigma_{2}$ \\
\hline 5 & $0 \quad 0$ & $2+2$ & $\Sigma_{2} \times \Sigma_{2}$ \\
\hline 3 & $0-0$ & $3+1$ & $\Sigma_{3}$ \\
\hline 0 & $0-0-0$ & 4 & $\Sigma_{4}$ \\
\hline
\end{tabular}


The genera of the curves are computed using a formula from [Mc, pp. 55-56]. The degrees of the coverings can be read off from the orders of the corresponding subgroups. The ramification index of each covering is readily computed using the Riemann-Hurwitz formula.

\begin{tabular}{ccc}
$\frac{\text { covering }}{31 \rightarrow 13}$ & $\frac{\text { degree }}{2}$ & ramification index \\
\cline { 2 - 2 } $13 \rightarrow 5$ & 2 & 8 \\
$13 \rightarrow 3$ & 3 & 12 \\
$5 \rightarrow 0$ & 6 & 20 \\
$3 \rightarrow 0$ & 4 & 12
\end{tabular}

The spectral curves in the lattice are, of course, actually isomorphism classes of spectral curves. However, if the specific curves of genera 3 and 5 of [Mc, Sect. 6] are taken, one gets an embedding (genus 13 ) $\rightarrow$ (genus 3 ) $\times$ (genus 5) and one can check by considering the corresponding stabilizer subgroups that the genus 13 curve embedded this way is the graph of the correspondence between the genus 3 and the genus 5 curves which is analyzed in detail in [Mc, Sect. 6].

\section{References}

[AvM1] Adler, M., van Moerbeke, P.: Completely Integrable Systems, Euclidean Lie Algebras, and Curves. Adv. Math. 38, 267-317 (1980)

[AvM2] Adler, M., van Moerbeke, P.: Linearization of Hamiltonian Systems, Jacobi Varieties, and Representation Theory. Adv. Math. 38, 318-379 (1980)

[BRS] Bobenko, A.I., Reyman, A.G., Semenov-Tian-Shansky, M.A.: Commun. Math. Phys. 122, 321-354 (1989)

[Bo] Bogoyavlensky, O.I.: On Perturbations of the Periodic Toda Lattice. Commun. Math. Phys. 51, 201-209 (1976)

[BMP] Bremner, M.R., Moody, R.V., Patera, J.: Tables of Dominant Weight Multiplicities for Representations of Simple Lie Algebras. Pure and Applied Mathematics. New York, NY: Marcel Dekker 1985

[B] Brieskorn, E.: Die Fundamentalgruppe des Raumes der regulären Orbits einer endlichen komplexen Spiegelungsgruppe. Invent. Math. 12, 57-61 (1971)

[F] Forster, O.: Lectures on Riemann Surfaces. Berlin, Heidelberg, New York: Springer 1977

[GrRe] Grauert, H., Remmert, R.: Komplex Räume. Math. Ann. 136, 245-318 (1958)

[G] Griffiths, P.A.: Linearizing flows and a cohomological interpretation of Lax equations. Am. J. Math. 107 (1985)

[GR] Gunning, R.C., Rossi, H.: Analytic functions of several complex variables. Englewood Cliffs, NJ: Prentice-Hall 1965

[Hart] Hartshorne, R.: Algebraic geometry. GTM, Berlin, Heidelberg, New York: Springer 1977

[H1] Humphreys, J.E.: Linear algebraic groups. GTM, Berlin, Heidelberg, New York: Springer 1975

[H2] Humphreys, J.E.: Introduction to Lie algebras and representation theory. GTM, Berlin, Heidelberg, New York: Springer

[K] Kanev, V. Spectral curves, simple Lie algebras, and Prym-Tjurin Varieties. Proc. Symp. Pure Math. 49, 627-645 (1989)

[Kr] Krichever, I.M. Elliptic Solutions of the Kadomtsev-Petviashvili Equation and Integrable Systems of Particles. Funct. Anal. Appl. 14, 282-290 (1980)

[M] Milne, J.S.: Étale Cohomology. Princeton, N.J.: Princeton University Press 1980 
[Mc] McDaniel, A.: Representations of $s l(n, \mathbf{C})$ and the Toda lattice. Duke Math. J. 56, 47-99 (1988)

[MS] McDaniel, A., Smolinsky, L.: The topology of some spaces from Hamiltonian mechanics. Proc. of the KAIST mathematics workshop 5, 137-142 (1990)

[OP] Olshanetsky, M.A., Perelomov, A.M.: Classical Integrable Systems Related to Lie Algebras. Phys. Rep. 71, 313-400 (1981)

[T] Toda, M.: Theory of Nonlinear Lattices. SSS, Berlin, Heidelberg, New York: Springer 1981

[vM] van Moerbeke, P.: The Spectrum of Jacobi Matrices. Invent. Math. 37, 45-81 (1976)

[vMM] van Moerbeke, P., Mumford, D.: The Spectrum of Difference Operators and Algebraic Curves. Acta Math. 143, 93-154 (1979)

[ZM] Zakharov, V.E., Mikhailov, A.V.: Method of the Inverse Scattering Problem with Spectral Parameter on an Algebraic Curve. Funct. Anal. Appl. 17, 247-251 (1983)

Communicated by M. Herman 